\title{
DSR Routing Protocol in Wireless Ad-hoc Networks: Drop Analysis
}

\author{
Rakesh Poonia \\ Department of Computer \\ Applications, \\ Engineering College \\ Bikaner
}

\author{
Amit Kumar Sanghi \\ Department of Computer \\ Applications, \\ Engineering College \\ Bikaner
}

\author{
Dr. Dharm Singh \\ College of Technology \\ and Engineering, \\ MPUAT, Udaipur
}

\begin{abstract}
A Mobile Ad Hoc Network (MANET) is a network consisting of a collection of nodes capable of communicating with each other without aid from a network infrastructure. Each node participating in the network works both as host and a router and must therefore is willing to forward packets for other nodes. For this purpose, a routing protocol is needed. The most important characteristics of MANET is the dynamic topology, nodes can change position dynamically therefore a need of a routing protocol that quickly adapts to topology changes. In this paper for experimental purpose, Investigators considered $150 \mathrm{~m} \mathrm{x}$ $150 \mathrm{~m}, 250 \mathrm{~m} \times 250 \mathrm{~m}, 350 \mathrm{~m} \times 350 \mathrm{~m}, 450 \mathrm{~m} \times 450 \mathrm{~m}, 550 \mathrm{~m}$ $\mathrm{x} 550 \mathrm{~m}, 650 \mathrm{~m} \times 650 \mathrm{~m} \& 750 \mathrm{~m} \times 750 \mathrm{~m}$ terrain area and illustrate the Drop packet analysis using DSR protocol parameters for wireless network scenario. The Dynamic Source Routing protocol, a simple as well as an efficient routing protocol is designed particularly for use in multi-hop wireless ad hoc networks, allows the network to be entirely self-organizing and self-configuring, without the requirement of any presented network infrastructure or the administration. All aspects of the protocol work entirely on-demand, permitting the routing packet overhead to scale automatically to only which needed to respond to various changes in the different routes currently in use.
\end{abstract}

Keywords: Protocols, Drop, MANET, network, DSR, Wireless

\section{INTRODUCTION}

Wireless ad hoc network is a collection of autonomous mobile nodes that communicate with each other over wireless links. Such networks are expected to play increasingly important role in future organizations, University, civilian and military setting, being useful for providing communication support where no fixed infrastructure exists or the deployment of a fixed infrastructure is not economically profitable and movement of communicating parties is possible. Due to the limited transmission range of wireless network interfaces, multiple networks "hops" may be needed for one node to exchange data with another across the network. A node can move anytime in an ad hoc scenario and, as a result, such as base stations, mobile hosts need to operate as routers in order to maintain the information and the network connectivity.

In such a network, each mobile node operates not only as a host but also as router, forwarding packets for other mobile nodes in the network that may not be within direct wireless transmission range of each other [1]. The idea of ad-hoc networking is sometimes also called infrastructure less networking since the mobile nodes in the dynamically establish routing among themselves to form their own network "on the fly". Major challenges in mobile ad hoc networks are routing of packets with frequently mobile nodes movement, there are resource issues like power and storage and also wireless communication issues. As mobile ad hoc network consists of wireless hosts which may move very often. Movement of hosts results in a change of routes. In this paper, Investigator compares the Packet Drop DSR (Dynamic Source Routing) Protocol

The Dynamic Source Routing protocol (DSR) [2] is a simple as well as efficient routing protocol which is designed specifically for the use in different multi-hop wireless ad hoc networks of various mobile nodes. All the network nodes cooperate to forward multiple packets for each other for allowing communication over a lot of "hops" between nodes not directly within the wireless transmission range of one another. As all the mobile nodes in the network may join or leave the network through movement, and as the wireless transmission conditions such as sources of interference change and hence the routing is automatically determined as well as maintained by the DSR routing protocol. Since the number or the sequence of intermediate hops required to reach any destination may alter at any time, the resulting network topology may be quite affluent and rapidly changing.

The DSR protocol permits various nodes to dynamically find out a source route across some multiple network hops to any destination in mobile ad hoc network. Each data packet which is sent carries in its header the complete as well as ordered list of different nodes through which the packet must pass, allowing the packet routing to be insignificantly loop-free and avoiding the requirement for all up-to-date routing information in the midway nodes through which the packet is forwarded. So by including this source route in the header of each data packet, some other nodes forwarding or overhearing any of these packets may also simply cache this routing information for the future use.

The designing of DSR is to create a routing protocol which had very low overhead and be able to react quickly to various changes in the network, providing a highly reactive service to ensure a successful delivery of different data packets in spite of the movement of nodes or some other changes in the network conditions.

The design of DSR incorporates two mechanisms which make up various operations of DSR, Route Discovery and Route Maintenance.[3] DSR is used in different supporting heterogeneous networks as well as interconnecting to the Internet and it supports for the routing of multicast packets in ad hoc networks.

\section{ROUTING PROTOCOL}

Before a packet reaches to its final destination, it may be necessary to a packet to hop several hops (also known as multi hop) as multi hoping is required for this routing protocol. The routing protocol has two main functions, the selection of routes for various source-destination pairs as well as the delivery of different messages to their correct destination. The second 
method is conceptually straight forward using a variety of protocols and data structures (routing tables).

Routing Protocols can be divided into various categories depending on their properties

- Centralized Vs Distributed

- Static Vs Adaptive

- Reactive Vs Proactive

One way to categorize the routing protocols is to divide them into centralized and distributed algorithms. In centralize algorithms, all route choices are made to a central node, while in distributed algorithms, the computation of routes is share among the network node. Another classification of routing protocols relates to whether they change routes in response to the traffic input patterns. In static algorithm, the route use is sourcedestination pairs are fixed regardless of traffic conditions [4]. It can only change in response to a node or link failure. This type of algorithm cannot achieve high throughput under a broad variety of traffic input patterns. Most major packet networks uses some form of adaptive routing where the routes use to route between source-destination pairs may change in response to congestion.

A third classification that is more related to ad hoc network is to classify the routing algorithms as either proactive or reactive. Proactive protocols attempt to continuously evaluate the routes within the network, so that when a packet needs to be forwarded, the route is already known and can be immediately used. The family of distance vector protocols is an example of proactive schemes. Reactive protocols, on the other hand, invoke a route determination procedure on demand only. Thus, when a route is needed, some sort of global search procedure is employed. The family of classical flooding algorithms belongs to the reactive group. Proactive schemes have the advantage that when the route is needed, the delay before actual packets can be sending is very small. On the other hand reactive schemes needs time to converge to a steady state. This can cause problem if the topology is changing frequently.

\subsection{DSR}

Dynamic Source Routing Protocol is a reactive routing protocol and is called on demand routing protocol [5]. It is a source routing protocol that is why it is a simple and an efficient protocol. It can be used in multi hop wireless ad hoc networks. The DSR network is totally self organizing and self configuring. The protocols is just compose of two mechanisms i.e. route discovery and route maintenance.

The DSR regularly updates its route cache for the sake of new available easy routes. If some new available routes were found the node will directs the packet to that route. The packet has to know about the route direction. So the information about the route was set in the packet to reach its destination from its sender. This information was kept in the packet to avoid periodic findings it has the capability to find out its route by this way. DSR has two basic mechanisms for its operation i.e. route discovery and route maintenance. In route discovery, it has two messages i.e. route request (RREQ) and route reply (RREP). When a node wishes to send a message to a specific destination, it broadcast the RREQ packet in the network. The neighbor nodes in the broadcast range receive this RREQ message and add their own address and again rebroadcast it in the network. This RREQ message if reached to the destination, so that is the route to the specific destination. In the case if the message did not reached to the destination then the node which received the RREQ packet will look that previously a route used for the specific destination or not.
Each node maintains its route cache which is kept in the memory for the discovered route. The node will check its route cache for the desired destination before rebroadcasting the RREQ message. By maintaining the route cache at every node in the network, it reduces the memory overhead which is generated by the route discovery procedure. If a route is found in that node route cache then it will not rebroadcast the RREQ in the whole network. So it will forward the RREQ message to the destination node. The first message reached to the destination has full information about the route. That node will send a RREP packet to the sender having complete route information.

The DSR protocol [6] is composed of following two mechanisms which work together to allow the discovery as well as the maintenance of source routes in the ad hoc network:

$>$ Route Discovery is a mechanism in which a node $\mathrm{S}$ which is wishing to send a packet to a destination node $\mathrm{D}$ obtains a source route to $\mathrm{D}$. The route Discovery is used only when $\mathrm{S}$ tries to send a packet to $\mathrm{D}$ but does not already know a route to $\mathrm{D}$.

$>$ Route Maintenance is another mechanism by which a node $\mathrm{S}$ is capable to detect, while using a source route to $\mathrm{D}$, while the network topology has been changed such that it can no longer utilize its route to $\mathrm{D}$ as a link along with the route no longer works. When Route Maintenance specifies a source route has been broken, $\mathrm{S}$ can attempt to employ any other route which happens to know to D, or may invoke the Route Discovery again to discover a new route. The Route Maintenance is used only when $\mathrm{S}$ is really sending packets to D.

Route Discovery as well as Route Maintenance each operates completely on demand. Particularly, unlike other protocols, DSR does not require periodic packets of any kind at any level within the network. For example, the DSR does not employ any kind of periodic routing advertisement, the link status sensing or neighbor detection packets, and it does not rely on all these functions from any underlying protocols within the network. This completely on-demand behavior as well as the lack of periodic activity simply allows the number of overhead packets to scale all the way down to zero, in case when all nodes are about stationary with respect to each other and all the routes required for current communication have already been discovered.

As all the nodes begin to move more or as the communication patterns change, routing packet overhead of DSR routinely scales to only which needed to track various routes currently in use. In response to a single Route Discovery, a node may learn as well as cache multiple routes to any destination. This permits the reaction to several routing changes to be much more rapid as a node having multiple routes to a destination may try another cached route in case the one it has been using should not succeed. The caching of numerous routes also avoids the overhead of requirement to perform a novel Route Discovery every time a route in use breaks. The operation of Route Discovery as well as Route Maintenance in DSR, are designed to allocate uni-directional links as well as asymmetric routes to be easily supported. In wireless networks, it is probable that a link between any two nodes may not work similarly well in both directions, due to differing antenna or the propagation patterns or various sources of interference.

DSR allows different uni-directional links to be used when essential, hence improving overall performance as well as the network connectivity in the system [7]. DSR also supports an internetworking between different types of wireless networks and allowing the source route to be composed of hops over a grouping of any types of networks available. For instance, a 
number of nodes in the ad hoc network may have only shortrange radios, even as other nodes have both short-range as well as long-range radios; a combination of all these nodes jointly can be considered by DSR as a single ad hoc network. Additionally, the routing of DSR has been incorporated into standard Internet routing, where a "gateway" node connected to the Internet also makes participation in the ad hoc network routing protocols and has been integrated into Mobile IP routing.

\section{SIMULATION SETUP}

In this paper for experimental purposes, Investigators considered ns2 [8] simulator. NS, a network simulator which was developed by Berkeley University, is used for simulation purposes. NS2 helps in developing tools for simulation results display, analysis and converters that convert network topologies to NS formats. NS2 is written in C++ and OTcl (Object-oriented tool command language).

The simulation parameters used for the first set of experiment-1 are shown in Table I and find out that total no of drop are 851.

Table I: Simulation Parameter

\begin{tabular}{|l|l|}
\hline Parameter & Value \\
\hline Terrain area & $150 \mathrm{~m} \times 150 \mathrm{~m}$ \\
\hline Routing Protocol & DSR \\
\hline No of Nodes & 20 \\
\hline Pause Time & $20 \mathrm{Sec}$ \\
\hline Maximum Speed & $20 \mathrm{M} / \mathrm{sec}$ \\
\hline Simulation Time & $1000 \mathrm{Sec}$ \\
\hline Maximum connection & 10 \\
\hline Seed value & 0.0 \\
\hline Sending rate & 3 \\
\hline
\end{tabular}

In experiment 2, we have set all parameter same as experiment 1 except the Terrain area. In this experiment we have taken the terrain area: $250 \mathrm{~m} \times 250 \mathrm{~m}$ and find out that total no of drops are 937

In experiment 3 also, we have set all parameter same as in previous except the Terrain area. In this experiment we have increase the terrain area: $350 \mathrm{~m} \times 350 \mathrm{~m}$ and find out that total no of drops are 1107

In experiment 4 also, we have set all parameter same as in previous experiment except the Terrain area. In this experiment we have taken the terrain area: $450 \mathrm{~m} \times 450 \mathrm{~m}$ and find out that total no of drops are 1504

In experiment 5 also, we have set all parameter same as in previous experiment except the Terrain area. In this experiment we have taken the terrain area: $550 \mathrm{~m} \times 550 \mathrm{~m}$ and find out that total no of drops are 1624

In experiment 6 also, we have set all parameter same as in previous experiment except the Terrain area. In this experiment we have taken the terrain area: $650 \mathrm{~m} \times 650 \mathrm{~m}$ and find out that total no of drops are 1477, which are less than in previous experiments.

In experiment 7 also, we have set all parameter same as in previous experiment except the Terrain area. In this experiment we have taken the terrain area: $750 \mathrm{~m} \times 750 \mathrm{~m}$ and find out that total no of drops are 12507, which are less than in previous experiments.
Thus if we observe terrain area and no of drops, that shown in Table II.

Table II: Packet drop

\begin{tabular}{|l|l|}
\hline Terrain Area & No of drops \\
\hline $150 \mathrm{~m} \times 150 \mathrm{~m}$ & 851 \\
\hline $250 \mathrm{~m} \times 250 \mathrm{~m}$ & 937 \\
\hline $350 \mathrm{~m} \times 350 \mathrm{~m}$ & 1107 \\
\hline $450 \mathrm{~m} \times 450 \mathrm{~m}$ & 1504 \\
\hline $550 \mathrm{~m} \times 550 \mathrm{~m}$ & 1624 \\
\hline $650 \mathrm{~m} \times 650 \mathrm{~m}$ & 1477 \\
\hline $750 \mathrm{~m} \times 750 \mathrm{~m}$ & 1250 \\
\hline
\end{tabular}

As we analyze Terrain Area and No of drops we find that if the Terrain Area is $150 \mathrm{~m} \times 150 \mathrm{~m}$ no of packet drops are 851 , in terrain area $250 \mathrm{~m} \times 250 \mathrm{~m}$ packets drops are 937, in terrain area $350 \mathrm{~m} \times 350 \mathrm{~m}$ drops are 1107 , in terrain area $450 \mathrm{~m} \times 450 \mathrm{~m}$ drops are 1504 , in terrain area $550 \mathrm{~m} \times 550 \mathrm{~m}$ drops are 1624 . But now if we increase the terrain area i.e. $650 \mathrm{~m} \times 650 \mathrm{~m}$ drops decreases and comes to 1477 and as we increase terrain area more i.e. $750 \mathrm{~m} \times 750 \mathrm{~m}$ no of drops decreases and comes to 1250 only. This trend can also be visualized in the Figure 1.



Figure 1: Terrain area vs. Packet drop

\section{CONCLUSION}

In this paper we have deal with DSR routing algorithm in mobile ad hoc networks. We have focused on terrain area and no of drops in DSR routing algorithm. Thus we promptly able to say that as the terrain area is less $(150 \mathrm{~m} \times 150 \mathrm{~m})$ no of drops are also less (850) and the terrain area increases (550 x 550) no of drops are also increases (1624). But if we increase more terrain area i.e beyond $550 \times 550$ to $650 \times 650$ no of drops become less i.e 1477 . And as we have increase terrain area $750 \times 750$ no of drops become less i.e 1250 .

\section{REFERENCE}

[1] Dimitri Bertsek and Robert Gallager, "Data Networks - 2nd ed”. Prentice Hall, New Jersey, ISBN 0-13-200916-1.

[2] D. Maltz D. Johnson, Y. Hu. The dynamic source routing protocol (dsr) for mobile ad hoc networks for ipv4. February 2007. URL http://tools.ietf.org/html/ rfc4728

[3] J.A.G. Garrido, Routing in Wireless Mobile Ad Hoc Networks: Adaptive Cache Mechanism for Dynamic Source Routing, Saarbrucken: VDM, 2008. 
[4] Yoo Y, Ahn S, Agrawal DP, "Impact of a simple load balancing approach and an incentive-based scheme on MANET performance" Journal of Parallel and Distributed Computing, Volume: 70 Issue: 2, Pages: 71-83, FEB 2010.

[5] Josh Broch, David A. Maltz, David B. Johnson, Yih-Chun $\mathrm{Hu}$, and Jorjeta Jetcheva. A Performance Comparison of Multi-Hop Wireless Ad Hoc Network Routing Protocols. In Proceedings of the Fourth Annual ACM/IEEE International Conference on Mobile Computing and Networking (MobiCom'98), pages 85-97, Dallas, TX, October1998 ACM.
[6] David B. Johnson, David A. Maltz and Yih-Chun Hu, "The Dynamic Source Routing Protocol for Mobile Ad Hoc Networks (DSR)", RFC 4728, February 2007.

[7] http://www.networksorcery.com/enp/protocol/dsr.htm-Dynamic source routing protocol (DSR)

[8] Information Sciences Institute, "The Network Simulator - ns2", June 2004, <http://www.isi.edu/nsnam/ns/> 